\title{
Radiofrequency Heat-Enhanced Chemotherapy for Breast Cancer: Towards Interventional Molecular Image-Guided Chemotherapy
}

\author{
Yurong Zhou ${ }^{1}$, Guocan Han ${ }^{1}$, Yue Wang ${ }^{1}$, Xi Hu${ }^{1}$, Zhiming Li1, Lumin Chen ${ }^{1}$, Weixian Bai ${ }^{1}$, Jingfeng Luo', \\ Yajing Zhang1, Jihong Sun ${ }^{1 凶}$, Xiaoming Yang1,2凶 \\ 1. Department of Radiology, Sir Run Run Shaw Hospital, Zhejiang University School of Medicine, Hangzhou, Zhejiang, China. \\ 2. Image-Guided Bio-Molecular Intervention Research, Department of Radiology, University of Washington School of Medicine, Seattle, \\ Washington, USA.
}

\begin{abstract}
$\triangle$ Corresponding author: Professor Xiaoming Yang, Image-Guided Bio-Molecular Intervention Research, Department of Radiology, University of Washington School of Medicine, 815 Mercer Street, Room S470, Seattle, WA 98109, USA. Tel: 206-685-6967; E-mail: xmyang@uw.edu OR Jihong Sun MD. PhD. Co-corresponding author, Department of Radiology, Sir Run Run Shaw Hospital, Zhejiang University School of Medicine, 3 East Qingchun Road, Hangzhou, Zhejiang, China, 310016. Tel: (0571)86006764; E-mail:braversun@sina.com.
\end{abstract}

(C) Ivyspring International Publisher. This is an open-access article distributed under the terms of the Creative Commons License (http://creativecommons.org/ licenses/by-nc-nd/3.0/). Reproduction is permitted for personal, noncommercial use, provided that the article is in whole, unmodified, and properly cited.

Received: 2014.06.29; Accepted: 2014.08.08; Published: 2014.09.10

\begin{abstract}
Breast cancer is the most common malignancy in women worldwide. Recent developments in minimally invasive interventional radiology techniques have significantly improved breast cancer treatment. This study aimed to develop a novel technique for the local management of breast cancers using radiofrequency heat (RFH). We performed both in vitro experiments using human breast cancer cells and in vivo validation in xenograft animal models with magnetic resonance imaging (MRI) and pathological correlation to investigate the feasibility of our approach. Four treatment groups, including (I) no treatment (control), (2) RFH-only, (3) chemo (doxorubicin)-only, and (4) combination therapy with both doxorubicin and RFH, were conducted in each experiment. In vitro combination therapy significantly decreased breast cancer cell proliferation while increased their apoptosis index compared to the other three groups. MRI demonstrated a significant tumor size reduction in animals treated with combination therapy compared to those receiving other treatments in vivo. Such result was further confirmed by pathological examination. In conclusion, our findings suggests that RFH can enhance the therapeutic efficiency of doxorubicin on breast cancers, thus establishing the basis for future development of interventional molecular image-guided local chemotherapy for breast malignancies.
\end{abstract}

Key words: breast cancer; radiofrequency; doxorubicin; MRI; hyperthermia.

\section{Introduction}

Breast cancer is the most common malignancy in women with a steadily increasing incidence worldwide. It is estimated that 235,030 patients will be diagnosed with invasive breast cancer and 40,430 will die of the disease in the United States in 2014 [1]. Although systemic chemotherapy is one of the main therapeutic strategies for breast cancer, there is no guarantee that sufficient concentration of chemotherapeutic agents would be delivered to the target tumor without causing toxicities to other vital organs [2]. In addition, previous studies have suggested that inefficient drug deposit at the target tumors via systemic administration significantly contribute to chemoresistance in cancer [3].

Recent studies have reported that hyperthermia at approximately $41{ }^{\circ} \mathrm{C}$ to $45^{\circ} \mathrm{C}$ can enhance the efficiency of chemotherapy in a variety of malignancies [4-7]. However, certain limitations, such as inade- 
quate devices for local heat delivery to the targets and lack of appropriate temperature monitoring at the targets, limit the application of hyperthermia-enhanced therapy for cancer in clinical practice $[8,9]$.

Minimally invasive interventional radiology techniques have greatly improved outcome of breast cancer treatment [10]. A combination of interventional radiology with locally delivered hyperthermia may therefore advance current chemotherapies. The present study aimed to develop a novel technique for local chemotherapy of breast cancer using interventional radiofrequency heat (RFH).

\section{Materials and Methods}

The present study included both in vitro establishment of the "proof-of-principle" that RFH enhanced chemotherapeutic efficiency in human breast cancer cells, and in vivo validation of interventional RFH-enhanced chemotherapy for breast cancer using xenograft animal models.

\section{In vitro experiments}

\section{Cells}

Human breast cancer cell (Bcap-37) were cultured in RPMI 1640 medium (Gibco-Life Technolo- gies, Mulgrave, Australia) adjusted to contain 1.5-g/L sodium bicarbonate, $2.5-\mathrm{g} / \mathrm{L}$ glucose, and $0.11-\mathrm{g} / \mathrm{L}$ sodium pyruvate supplemented with $10 \%$ fetal bovine serum (Gibco-Life Technologies). They were incubated at $37^{\circ} \mathrm{C}$ in a humidified atmosphere with $5 \%$ $\mathrm{CO}_{2}$.

\section{In vitro experimental set-up}

These breast cancer cells were seeded in each chamber of a Lab-Tek® 4-chamber cell culture slide (Nalge Nunc International, Rochester, NY, USA) at 4 $\times 10^{4}$ cells/chamber. The slice was placed in a $37^{\circ} \mathrm{C}$ water bath. A 0.032-inch magnetic resonance imaging-heating-guidewire (MRIHG) was attached under the bottom of chamber 4 of the 4-chamber cell culture slide, and then connected to a $2450-\mathrm{MHz}$ radiofrequency (RF) generator (GMP150, OPTHOS, Rockville, MD, USA) (Figure 1). When the RF generator was operated at 2-3 Watts through the MRIHG, the temperature in chamber 4 increased to approximately 42 ${ }^{\circ} \mathrm{C}$ from $37^{\circ} \mathrm{C}$, which created a heat gradient along the 4 chambers. The temperature of each chamber was recorded by a thermometer (Photon Control, Burnaby, Canada).

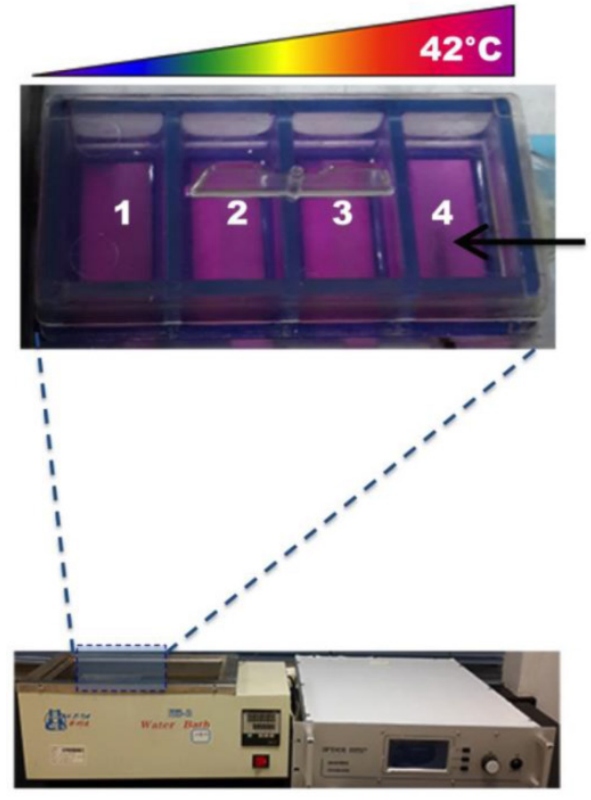

A
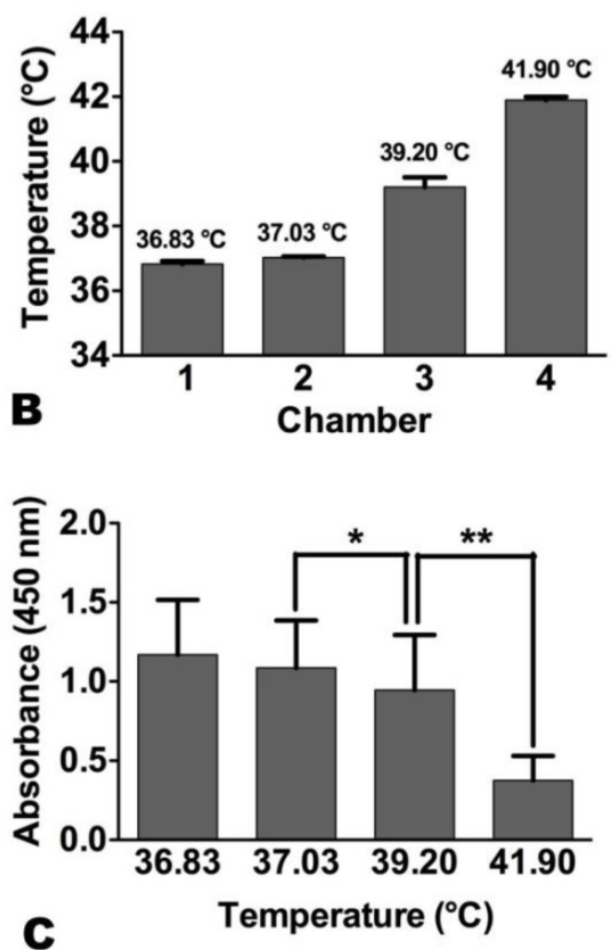

Figure I. (A) In vitro experimental set-up for radiofrequency heat (RFH) of human breast cancer cells. Cells are seeded in a 4 -chamber cell culture slide, which is placed in $37^{\circ} \mathrm{C}$ water bath. A 0.032 -inch magnetic resonance imaging-heating-guidewire (arrow) is positioned under the bottom of chamber 4 . (B) When the temperature reaching $42{ }^{\circ} \mathrm{C}$ in chamber 4 , a stable heat gradient is observed along the four chambers. (C) The proliferation of cells treated with $\mathrm{RFH}$ at $42{ }^{\circ} \mathrm{C}$ is significantly decreased compared to those treated at $37^{\circ} \mathrm{C}$ and $39^{\circ} \mathrm{C}(*, p<0.05 ; * *, p<0.01)$. 


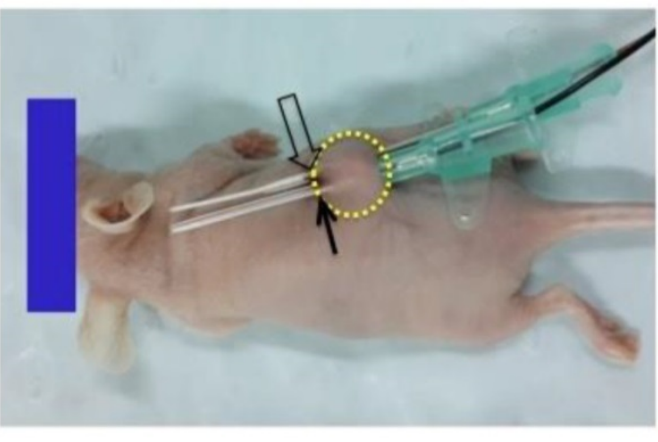

A

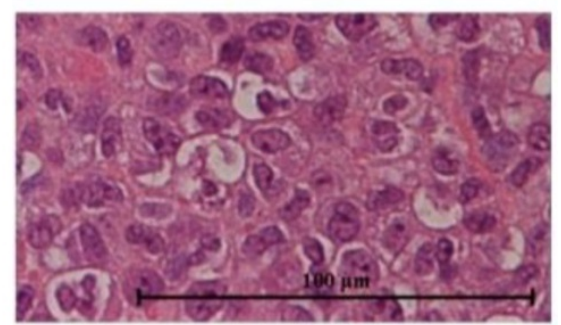

B

Figure 2. (A) In vivo experimental set-up for radiofrequency heat (RFH) of a xenograft tumor (circle) implanted in a nude mouse. Trans-tumor insertion of a 0.032 -inch magnetic resonance imaging-heating-guidewire (MRIHG) is performed (open arrow). The radiofrequency-heated tumor is maintained at $42^{\circ} \mathrm{C}$ by instantly measuring the temperature with a micro-figure thermometry wire (solid arrow), which is placed parallel to the MRIHG within the tumor. (B) Pathological study with hematoxylin and eosin staining confirms the successful generation of a breast xenograft tumor $(400 \times$ magnification).

For comparison of therapeutic effects among different RFH temperatures, the same amount of doxorubicin $(0.25 \mu \mathrm{M})$ was dripped into each of the four chambers while the bottom of chamber 4 was heated to $42^{\circ} \mathrm{C}$ for 20 minutes and that of chamber 1 remained at $37{ }^{\circ} \mathrm{C}$ (Figure 1). For comparison of therapeutic effects among different treatments, breast cancer cells were divided into groups: no treatment (control), RFH-only $\left(42{ }^{\circ} \mathrm{C}\right.$ for 20 minutes), chemo-only ( $0.25 \mu \mathrm{M}$ doxorubicin), and combination therapy (chemo plus RFH). The concentration of doxorubicin at $0.25 \mu \mathrm{M}$ was selected based on previous studies by other groups[11-13]. Cells were then cultured for 72 hours before different laboratory examinations, including cell proliferation assay and apoptosis assay, were conducted to examine and compare the effects of various treatments on breast cancer cells.

\section{Laboratory examinations}

Cell proliferation was assessed using the Cell Counting Kit-8 (CCK-8; Dojindo, Kamimashiki-gun Kumamoto, Japan) according to the manufacturer's instruction. Briefly, $100 \mu \mathrm{L}$ of CCK-8 solution was added into each chamber and incubated for 120 minutes. Then, cell proliferation was assessed by measuring the absorbance at $450 \mathrm{~nm}$ using a Universal Microplate Reader (BIO-TEK Instruments, Minneapolis, MN, USA). Cell apoptosis index was examined via staining with annexin $\mathrm{V}$-conjugated fluorescein isothiocynate (FITC) and propidium iodide (PI) as described in the annexin V-FITC apoptosis detection kit (Becton Dickinson Biosciences, San Diego, CA, USA), and flow cytometry analysis (Becton Dickinson FACScan, Mount View, VA, USA). Flow cytometry results were analyzed by Cell Quest Pro software (Becton Dickinson).

\section{In vivo experiments}

\section{In vivo experimental set-up}

The animal protocol was approved by our Institutional Animal Care and Use Committee. Female $\mathrm{nu} / \mathrm{nu}$ mice at 4-6 weeks of age were used to generate the tumor model. A suspension of $1 \times 10^{7} \mathrm{Bcap}-37$ cells in $100 \mu \mathrm{L}$ of phosphate-buffered saline (PBS) was injected subcutaneously into the unilateral back of each mouse to initiate a breast cancer mass (Figure 2). Within two weeks, the tumor masses grew to approximately $5 \mathrm{~mm}$ in diameter. A 0.032-inch MRIHG was inserted through the center of each tumor for local heating, while a $2.7-\mathrm{mm}$ micro-thermometry fiber was placed parallel to the MRIHG for instant measurement of the MRIHG-mediated RF heating at the target tumor (Figure 2).

\section{RFH-enhanced chemotherapy}

Twenty-four mice bearing human breast cancer xenograft tumors were randomly stratified to four study groups (6 mice per group) with receiving different intratumoral treatments: (i) PBS (control), (ii) RFH-only $\left(42{ }^{\circ} \mathrm{C}\right.$ for 20 minutes via the MRIHG), (iii) chemo-only (intratumoral injection of $10-\mathrm{mg} / \mathrm{kg}$ doxorubicin), or (iv) combination therapy (chemo plus RFH).

\section{MRI follow-up}

Mice were anesthetized by intraperitoneal delivery of $4 \%$ chloral hydrate $(0.01 \mathrm{~mL} / \mathrm{g})$ for MRI follow-up. MRI was performed using a 3.0-Tesla MR scanner (GE Healthcare Corporation, New York, USA) by placing the mouse into a $100 \mathrm{~mm}$-diameter micro-imaging coil. MRI was acquired before and at days 7 and 14 after treatment. T1-weighted images (T1WI) and $0.2-\mathrm{mmol} / \mathrm{kg}$ gadodiamide-enhanced T1WI (Omniscan, GE Healthcare) were acquired us- 
ing a rapid acquisition with OAx T1 550 Spin Echo sequence: $\mathrm{TR} / \mathrm{TE}=550 \mathrm{~ms} / 15 \mathrm{~ms}$, field of view $=8$ $\mathrm{cm}$, matrix $=256 \times 256$, section thickness $=1.5 \mathrm{~mm}$, intersection gap $=0.5 \mathrm{~mm}, \mathrm{NEX}=2$; and total scan time $=3$ minutes and 31 seconds.

\section{Pathological confirmation}

After satisfactory MRI, mice were euthanized by $5 \% \mathrm{CO}_{2}$ and the tumor masses were harvested. We then estimated the volume of each mass using the following formula: tumor volume $=($ length $\times$ width $\times$ width) $/ 2$ [14]. Owing to inevitable variation in tumor size at the beginning of treatment, relative tumor volume (RTV) was used for tumor growth comparison. RTV was calculated using the following formula: $R T V=T V_{n} / T V_{0}$, where $T V_{n}$ was the tumor volume at day $\mathrm{n}$ and $\mathrm{TV}_{0}$ was the tumor volume at day 0 [7].

The harvested tumor tissues were then fixed in $4 \%$ paraformaldehyde, embedded in paraffin, and sectioned at 5-mm slices. After de-waxing and hydration with a gradient ethanol series $(100 \%, 95 \%, 90 \%$, $80 \%$, and $70 \%$ ), tissue slices were stained with the in situ Cell Death Detection Kit Fluorescein (Roche, Basel, Switzerland) and then exposed to freshly-prepared proteinase $\mathrm{K}$ working solution for 15-30 minutes at $37{ }^{\circ} \mathrm{C} \quad(10-20 \mu \mathrm{g} / \mathrm{mL}$ in $10 \mathrm{mM}$ Tris/ $\mathrm{HCl}, \mathrm{pH}$ 7.4-8). After washing with PBS, slices were incubated in $50 \mathrm{~mL}$ of TUNEL (terminal deoxynucleotidyl transferase-mediated nick end-labeling) reaction mixture for 60 minutes in dark and humidified environment. They were again washed with PBS and sealed with Prolong Gold Antifade Reagent after staining with 4', 6-diamidino-2-phenylindole dihydrochloride (DAPI; Invitrogen, Carlsbad, USA) overnight. All slides were examined with a fluorescent microscope (Leica, Solms, Germany). The number of apoptotic cells was counted by Image-Pro Plus 6 software (Media Cybernetics, Rockville, MD, USA).

\section{Statistical analysis}

Statistical analysis was performed using SPSS (IBM, Armonk, New York, USA). Data were presented as the mean \pm standard deviation. One-way analysis of variance was performed to compare the average cell proliferation rate, cell apoptosis index, tumor volume, and RTV. Differences among the four study groups were considered statistically significant at $p$ $<0.05$.

\section{Results}

\section{RFH-enhanced chemotherapeutic efficiency in breast cancer cells}

In vitro combination therapy significantly decreased breast cancer cell proliferation $(0.62 \pm 0.04$ vs. $1.19 \pm 0.02$ vs. $1.00 \pm 0.07$ vs. $0.71 \pm 0.07, p<0.05)$ while increased their apoptosis index $(55.37 \pm 13.99 \%$ vs. $1.61 \pm 0.53 \%$ vs. $3.32 \pm 0.61 \%$ vs. $43.32 \pm 15.47 \%, p<$ $0.05)$, compared to the other three treatment groups (controls, RFH-only, and chemo-only, respectively; Figure 3).

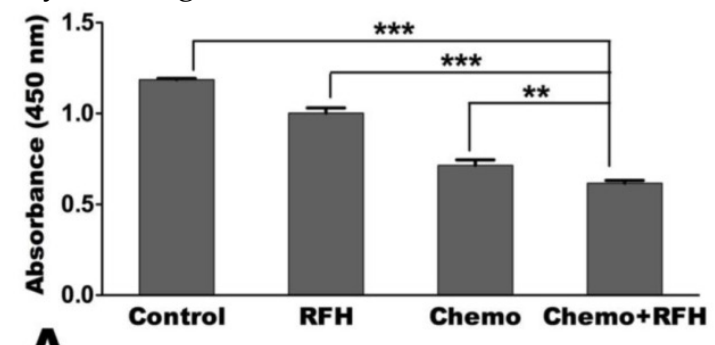

A

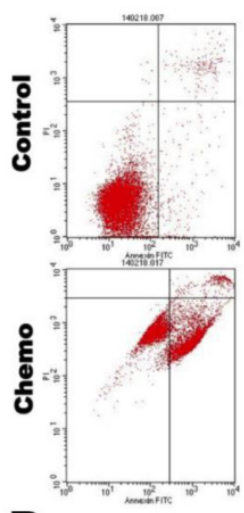

B

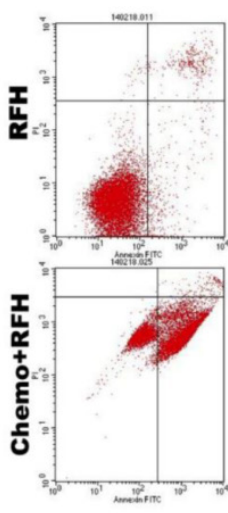

Figure 3. (A) Cell proliferation assay demonstrates that the proliferation of cells treated with combination therapy (chemo plus radiofrequency heat [RFH]) is significantly reduced compared to those receiving other treatments. (B) Representative results of apoptosis assay via flow cytometry. (C) Apoptosis assay shows a higher average apoptosis index in cells treated with combination therapy (chemo+RFH) compared to those receiving other treatments $(*, p<0.05 ; * *, p<0.01 ; * * *, p<0.00 \mathrm{I})$. 


\section{RFH-enhanced chemotherapy for breast cancer xenograft models}

MRI demonstrated a significant tumor size reduction in mice treated with combination therapy compared to those receiving control, RFH-only, and chemo-only treatments in vivo (Figure 4 ). Such result was confirmed by subsequent pathological examination (Figure 5). The average RTV in the combination treatment group was significantly smaller than those

Week 0 of the control, RFH-only, and chemo-only groups $(0.86 \pm 0.50$ vs. $4.46 \pm 1.16$ vs. $2.85 \pm 1.54$ vs. $2.01 \pm 0.33$, respectively, $p<0.05$; Figure 5). The number of apoptosis cells and average apoptosis index in the combination therapy group were significantly higher than those in other three groups (37.02 $\pm 11.25 \%$ vs. $9.80 \pm 4.22 \%$ vs. $9.04 \pm 8.50 \%$ vs. $23.29 \pm 10.92 \%, p<$ 0.05 ; Figure 6).
Week 1

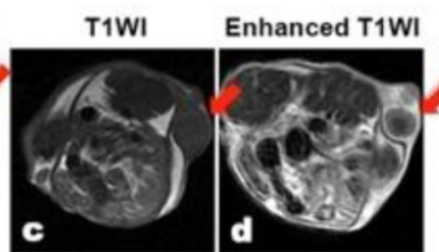

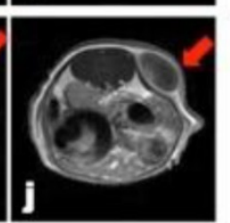

RFH
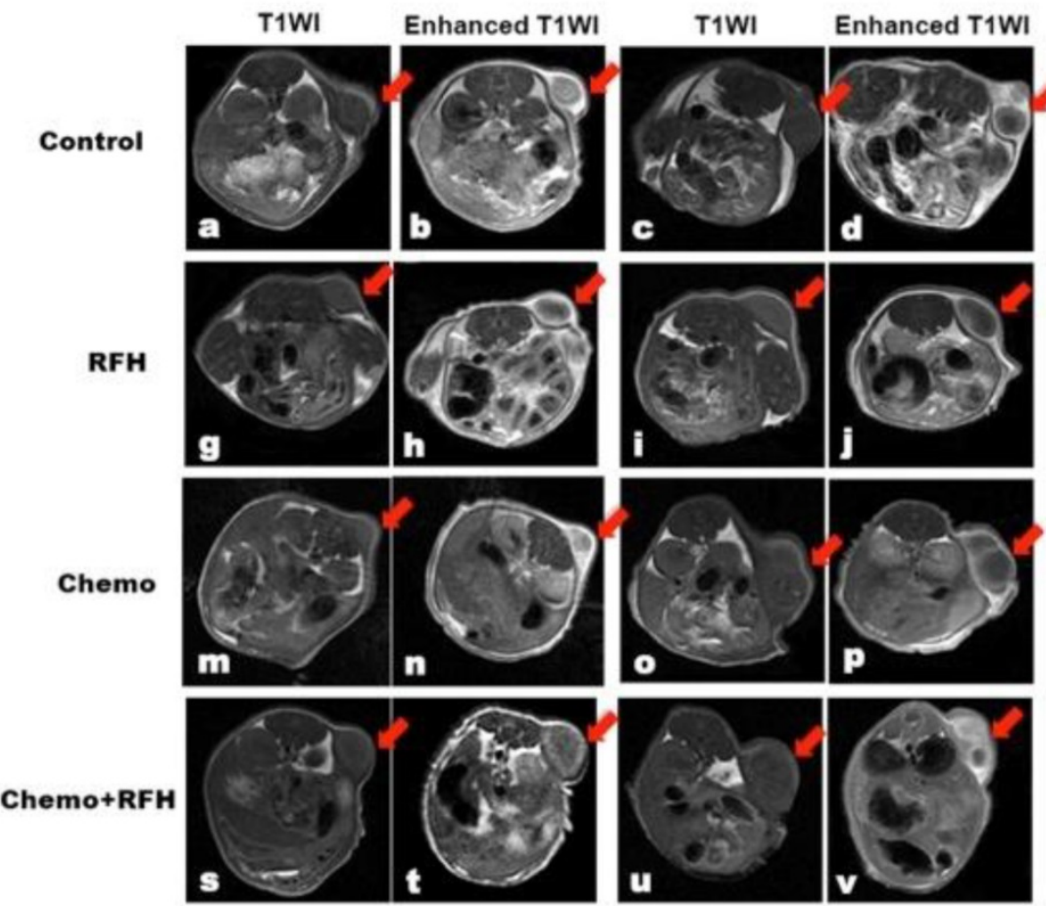

Figure 4. TI-weighted images (TIWI) of mice bearing breast cancer xenografts in all four treatment groups, demonstrating homogeneous hypointense tumor masses (arrows) on the animals' unilateral back. The tumor masses become hyperintense after intravenous administration of gadolinium (enhanced TIWI). The follow-up imaging of tumor growth at different time points shows that tumor size in the chemo plus radiofrequency heat (RFH) group ( $s-x)$ clearly decreases at week 2 after treatment (arrow on $x$ ), in comparison to those in the control (a-f), RFH-only ( $g-l)$, and chemo-only (m-r) groups.
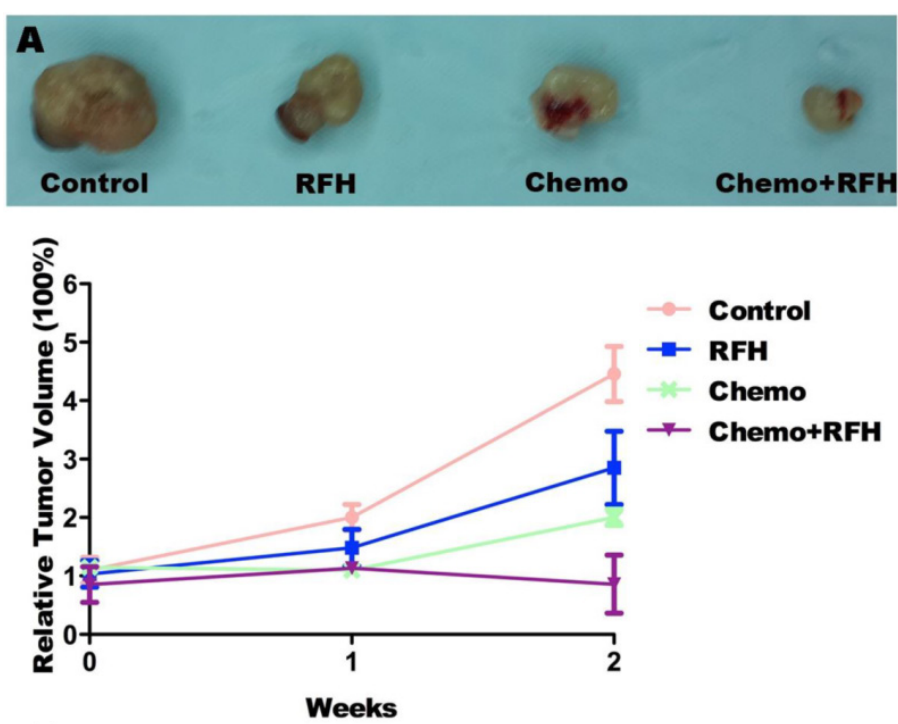

Figure 5. (A) Representative pathology of the tumor masses harvested from the four study groups further confirm the size reduction of tumor masses in mice receiving combination therapy with chemo plus radiofrequency heat (RFH). (B) Comparison of the relative tumor volume among the four treatment shows that RFH-enhanced chemotherapy significantly inhibits tumor growth at week 2 post-treatment.

\section{B}




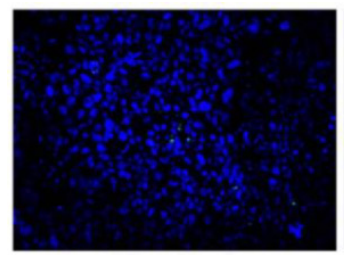

Control

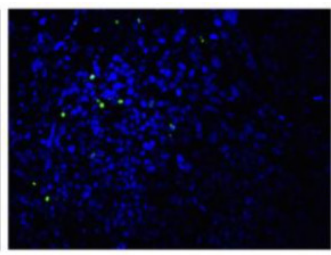

RFH

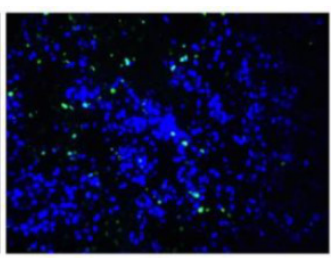

Chemo

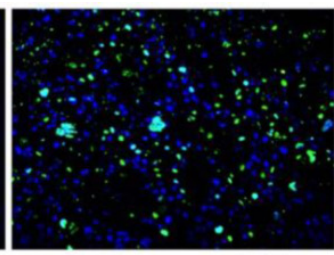

Chemo+RFH

A

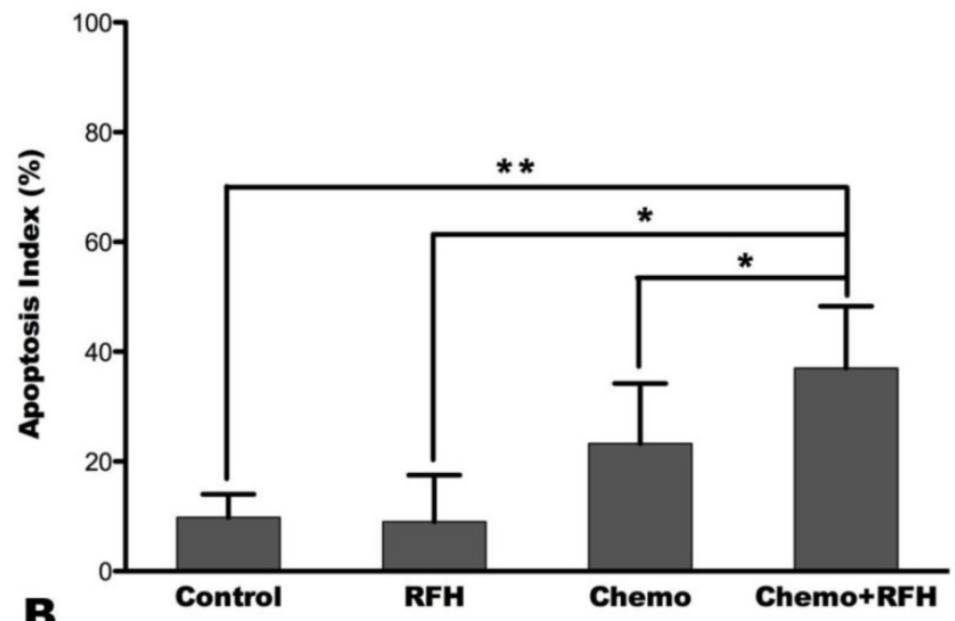

Figure 6. (A) Apoptosis assay demonstrates more apoptosis cells (green dots) in the combination therapy group (Chemo+RFH) than in other three groups, which is further confirmed by analysis of the apoptosis index, showing higher apoptosis index with combination treatment compared to other treatments $(B)(*, p<0.05$; $* *, p<0.0 \mathrm{I})$.

\section{Discussion}

Breast cancer is the most common malignancy in women worldwide. Although chemotherapy and hormone treatments have improved patient survival, ineffective chemotherapy remains a clinical problem owing to the disease's significant heterogeneity with different histologic components, gene-expression profiles, and mutational patterns [15]. Systemic administration of chemotherapy is conventional, but current technology does not guarantee sufficient accumulation of chemotherapeutic agents at the target tumors, whereas toxicities to other vital organs remain with systemic delivery $[2,16]$.

Recent developments of minimally-invasive interventional radiology technologies have significantly improved cancer management, including that of breast cancer $[17,18]$. Under the guidance of imaging, one can precisely place the interventional devices to the target tumors, thereby delivering highly concentrated therapeutics to the targets. Such local approaches can avoid the systemic administration of chemotherapeutic agents and thus minimize toxicity to other organs $[19,20]$.

In the present study, we attempted to overcome the disadvantages of systemic chemotherapy for breast cancer by developing a novel interventional therapeutic approach that combined the benefits of multiple modalities including RF technology, inter- ventional oncology, and chemotherapy. Our in vitro and in vivo results demonstrated that RFH could significantly improve the efficacy of chemotherapeutic agent (doxorubicin) in human breast cancer cells and that intratumorally delivered RFH could significantly enhance local chemotherapy for breast cancer. The mechanisms underlying RFH-enhanced chemotherapy might include heating to fracture tissue, increasing permeability of the cytoplasmic membrane, increasing cellular metabolism, and increasing the activity of heat shock proteins [21]. In addition, RFH itself may also impair the drug efflux ability of cancer cells. All these mechanisms facilitate the entrance of therapeutics into targeted tumor cells for effective destruction of tumor tissues, and thereby improve therapeutic outcome.

In addition, our novel technique potentially allows simultaneous intratumoral delivery of both chemotherapeutic agents and RFH, which might benefit conventional radiofrequency ablation (RFA) of tumors. RFA has become an important therapeutic tool for the treatment of unresectable tumors [22]. However, its application is limited in tumor masses that are in close proximity to normal structures prone to thermal injury, such as the vasculatures [23]. Furthermore, incomplete ablation often occurs at the tumor margins due to either decreased RFA heat by neighboring blood flows or irregularly shaped tumor masses being too large to be completely covered by 
the RFA electrode field [24]. Ultimately, such drawbacks of incomplete ablation often result in recurrences of RFA-treated tumors. A combination of RFH-enhanced local chemotherapy and intratumoral RFA may provide the opportunity of using RFA-associated peritumor hyperthermia to specifically enhance chemo-destruction of the tumor margins while avoiding RFA-related thermal injuries to the normal structures adjacent to the RFA-treated tumor masses [25, 26].

Further efforts are required to determine whether this novel combination therapy approach can increase endpoint survival and to validate this new technique in different tumor models with optimization of administered regimens. Bcap-37 cells were relatively fast growing breast cancer cells, and thus we had to limit our follow-up time up to two weeks after the treatments. This was because longer follow-up period would result in the xenograft tumor masses, especially in the control animal group, becoming more than ten percent of the body weight, which was not approved by our Institutional Animal Care and Use Committee. Thus, as a limitation, this study did not allow us to evaluate the long-term therapeutic effects with follow-up MRI.

In conclusion, the results of our study indicated that RFH might enhance the therapeutic efficacy of intratumorally delivered chemotherapy for breast cancer, thus establishing the groundwork for future development of interventional molecular image-guided local chemotherapy for breast malignancy using RF technology-integrated interventional oncology and chemotherapy.

\section{Acknowledgements}

This study was supported by National Basic Research Program of China (973 Program, 2014CB744505), National Institutes of Health grant (R01EB012467).

Program for National Science and Technology Major Project of China (2013ZX10002004-001-005), Qianjiang Talent Program of Zhejiang Province in China (2012R10027), Scientific Research Foundation of the Health Bureau of Zhejiang Province in China (WKJ2012-2-030), Fundamental Research Funds for the Central Universities (2012QNA7037) and SRF for ROCS, SEM.

\section{Abbreviations}

RFH: radiofrequency heat; MRI: magnetic resonance imaging; MRIHG: magnetic resonance imaging-heating-guidewire; RF: radiofrequency; CCK-8: Cell Counting Kit-8; FITC: fluorescein isothiocynate; PI: propidium iodide; PBS: phosphate-buffered saline; T1WI: T1-weighted images; RTV: relative tumor volume; TUNEL: terminal deoxynucleotidyl transferase-mediated nick end-labeling; DAPI: 4', 6-diamidino-2-phenylindole dihydrochloride; RFA: radiofrequency ablation.

\section{Conflict of Interests}

The authors have declared that no conflict of interest exists.

\section{References}

1. Siegel R, Ma J, Zou Z, et al. Cancer statistics, 2014. CA Cancer J clin. 2014; 64: 9-29.

2. Gradishar WJ, Anderson BO, Blair SL, et al. Breast cancer version 3.2014. J Natl Compr Canc Netw. 2014; 12: 542-90

3. Olive KP, Jacobetz MA, Davidson CJ, et al. Inhibition of Hedgehog signaling enhances delivery of chemotherapy in a mouse model of pancreatic cancer. Science. 2009; 324: 1457-61.

4. Coleman JA, Scardino PT. Targeted prostate cancer ablation: energy options. Curr Opin Urol. 2013; 23: 123-8.

5. Ahmed M, Moussa M, Goldberg SN. Synergy in cancer treatment between liposomal chemotherapeutics and thermal ablation. Chem Phys Lipids. 2012; 165: 424-37.

6. Zhang $\mathrm{T}$, Zhang $\mathrm{F}$, Meng $\mathrm{Y}$, et al. Diffusion-weighted MRI monitoring of pancreatic cancer response to radiofrequency heat-enhanced intratumor chemotherapy. NMR Biomed. 2013; 26: 1762-7.

7. Zhang $\mathrm{F}$, Le T, Wu X, et al. Intrabiliary RF heat-enhanced local chemotherapy of a cholangiocarcinoma cell line: monitoring with dual-modality imaging--preclinical study. Radiology. 2014; 270: 400-8

8. Pelicci PG, Dalton P, Orecchia R. Heating cancer stem cells to reduce tumor relapse. Breast Cancer Res. 2011; 13: 305.

9. Owusu RA, Abern MR, Inman BA. Hyperthermia as adjunct to intravesical chemotherapy for bladder cancer. Biomed Res Int. 2013; 2013:262313.

10. Zhang F, Li J, Meng Y, et al. Development of an intrabiliary MR imaging-monitored local agent delivery technique: a feasibility study in pigs. Radiology. 2012; 262: 846-52.

11. Zoli W, Ulivi P, Tesei A, et al. Addition of 5-fluorouracil to doxorubicin-paclitaxel sequence increases caspase-dependent apoptosis in breast cancer cell lines. Breast Cancer Res. 2005; 7: R681-9.

12. Fan $Y$, Ge N, Wang X, et al. Amplification and over-expression of MAP3K3 gene in human breast cancer promotes formation and survival of breast cancer cells. J Pathol. 2014; 232: 75-86.

13. Ghosh SK, Yigit MV, Uchida M, et al. Sequence-dependent combination therapy with doxorubicin and a survivin-specific small interfering RNA nanodrug demonstrates efficacy in models of adenocarcinoma. Int J Cancer. 2014; 134: 1758-66.

14. Naito S, von Eschenbach AC, Giavazzi R, et al. Growth and metastasis of tumor cells isolated from a human renal cell carcinoma implanted into different organs of nude mice. Cancer Res. 1986; 46: 4109-15.

15. Ignatiadis M, Sotiriou C. Luminal breast cancer: from biology to treatment. Nat Rev Clin Oncol. 2013; 10: 494-506.

16. Evangelista L, Mezzato C, Felloni G, et al. Current and future perspectives in diagnostic imaging as a guide to targeted/local therapies in breast cancer recurrence. Q J Nucl Med Mol Imaging. 2013; 57: 367-80.

17. Manenti G, Perretta T, Gaspari E, et al. Percutaneous local ablation of unifocal subclinical breast cancer: clinical experience and preliminary results of cryotherapy. Eur Radiol. 2011; 21: 2344-53.

18. Sridhar AN, Hughes-Hallett A, Mayer EK, et al. Image-guided robotic interventions for prostate cancer. Nat Rev Urol. 2013; 10: 452-62.

19. Ahmed M, Brace CL, Lee FT, Jr., et al. Principles of and advances in percutaneous ablation. Radiology. 2011; 258: 351-69.

20. Kim KW, Chow O, Parikh K, et al. Peritoneal carcinomatosis in patients with gastric cancer, and the role for surgical resection, cytoreductive surgery, and hyperthermic intraperitoneal chemotherapy. Am J Surg. 2014; 207: 78-83.

21. Madio DP, van Gelderen P, DesPres D, et al. On the feasibility of MRI-guided focused ultrasound for local induction of gene expression. J Magn Reson Imaging. 1998; 8: 101-4.

22. Qiu J, Chen S, Wu H. Long-term outcomes after hepatic resection combined with radiofrequency ablation for initially unresectable multiple and bilobar liver malignancies. J Surg Res. 2014; 188: 14-20.

23. Fonseca AZ, Santin S, Gomes LG, et al. Complications of radiofrequency ablation of hepatic tumors: Frequency and risk factors. World J Hepatol. 2014; 6: 107-13.

24. Huang HW. Influence of blood vessel on the thermal lesion formation during radiofrequency ablation for liver tumors. Med Phys. 2013; 40: 073303.

25. Wei Z, Ye X, Yang X, et al. Microwave Ablation in Combination with Chemotherapy for the Treatment of Advanced Non-Small Cell Lung Cancer. Cardiovasc Intervent Radiol. 2014; [Epub ahead of print].

26. Donati $\mathrm{M}$, Basile F. New trends in the multidisciplinary treatment of liver tumors. Future Oncol. 2013; 9: 1093-6. 


\section{Author biography}

Xiaoming Yang, MD, $\mathrm{PhD}$, is professor and director of Image-Guided Bio-Molecular Intervention Research at University of Washington School of Medicine, Seattle, WA, USA. He is also a professor at the Department of Radiology, Sir Run Run Shaw Hospital, Zhejiang University School of Medicine, China. He has coauthored more than 90 publications in many leading journals in the field of medical imaging, such as Radiology, Circulation, Nature Reviews Cardiology, Magnetic Resonance in Medicine, and Journal of Vascular and Interventional Radiology. The current research interests of Dr. Yang's laboratory include interventional radiology and molecular imaging.

Yurong Zhou, MD, obtained her MD from Tongji Medical College of Huazhong University of Science and Technology in 2004. She is currently a Ph.D. candidate at Zhejiang University School of Medicine under the supervision of Dr. Xiaoming Yang. Her research focuses on medical imaging. 